\title{
Systematic review and meta-analysis of the prognostic value of Narcotrend monitoring of different depths of anesthesia and different Bispectral Index (BIS) values for cognitive dysfunction after tumor surgery in elderly patients
}

\author{
Xinhua Shi ${ }^{1 \#}$, Xiangnan Chen ${ }^{2 \#}$, Jun $\mathrm{Ni}^{1}$, Yanqing Zhang ${ }^{1}$, Hui Liu ${ }^{3}$, Chuan $\mathrm{Xu}^{4}$, Hao Wang ${ }^{4}$ \\ ${ }^{1}$ Department of Anesthesiology, Nanjing Gaochun People's Hospital, Nanjing, China; ${ }^{2}$ Department of Anesthesiology, Guangdong Women \\ and Children Hospital, Guangzhou, China; ${ }^{3}$ Department of Cardiovascular Surgery, Linfen Central Hospital, Linfen, China; ${ }^{4}$ Department of \\ Anesthesiology, Linfen Central Hospital, Linfen, China \\ Contributions: (I) Conception and design: All authors; (II) Administrative support: H Wang; (III) Provision of study materials or patients: All authors; \\ (IV) Collection and assembly of data: All authors; (V) Data analysis and interpretation: X Shi, X Chen; (VI) Manuscript writing: All authors; \\ (VII) Final approval of manuscript: All authors. \\ \#These authors contributed equally to this work. \\ Correspondence to: Hao Wang. Associate Chief Physician, Department of Anesthesiology, Linfen Central Hospital, Jiefang West Road No. 17, Linfen \\ 041000, China. Email: wanghao55669@outlook.com.
}

Background: To study the effect of Narcotrend monitoring on the incidence of early postoperative cognitive dysfunction (POCD) under different Bispectral Index (BIS) conditions and the effect of different depths of anesthesia on the incidence of POCD.

Methods: We performed a literature search of the PubMed, Embase, OVID (database system made by Ovid Technologies, USA), Chinese Biomedical Literature Database (CBM), China National Knowledge Infrastructure (CNKI), VIP Chinese Sci-tech Journals Database, Wanfang Data, etc. from the date of establishment of the database until December 31, 2020.

Results: In the end, eighty articles were included, with a total of 714 patients. The meta-analysis results showed that four articles (255 patients) compared the state of deep anesthesia (BIS 30-40) with conventional anesthesia (BIS 40-60 earlier) after POCD. Also, the incidence of POCD on the first day after deep anesthesia [Narcotrend stage (NTS): negative correlation is currently the most appropriate egg description; $\mathrm{Nd}$ can subdivide the original electroencephalogram (EEG) into six stages 15 levels (Nd Sg, NTS), or A (state of wakefulness), state B0-B2 (sedated state), state C0-C2 (light anesthetic state), state D0-D2 (general anesthesia), state E0-E2 (deep anesthesia state), and state F0-F2 (burst suppression state)] was significantly lower than that of conventional anesthesia (NTS D0-D1) [odds ratio (OR) $=0.21,95 \%$ confidence interval (CI): $0.13-0.35, \mathrm{P}<0.00001]$. Moreover, the incidence of POCD in deep anesthesia (NTS E1) at 7 days after surgery was significantly lower than that of conventional anesthesia (NTS D0) (OR =0.45, 95\% CI: 0.23-0.91, $\mathrm{P}=0.03$ ), while the incidence of POCD 7 days after NTS D2 in conventional anesthesia was significantly lower than that of NTS D0 (OR =0.42, 95\% CI: 0.24-0.71, P=0.001).

Discussions: POCDs are thought to be the result of a combination of physical defects and precipitating factors in patients with their own physical impairments, and despite potential adverse effects, there is currently no consensus on the incidence of POCDs in patients with tumor, current risk factors, causes, and prevention strategies. Moreover, the level of evidence is low, and the deviation between different studies cannot be ruled out.

Keywords: Depth of anesthesia; Narcotrend monitoring; Bispectral Index value (BIS value); postoperative cognitive dysfunction (POCD); meta-analysis 
Submitted Nov 08, 2021. Accepted for publication Feb 14, 2022.

doi: $10.21037 / \mathrm{atm}-22-90$

View this article at: https://dx.doi.org/10.21037/atm-22-90

\section{Introduction}

Postoperative cognitive dysfunction (POCD) is a postoperative complication of the central nervous system (1). It is mainly manifested as differing degrees of obstacles such as memory, judgment, thinking, intelligence, orientation, etc., accompanied by a decline in social activity ability, and patients often experience anxiety, confusion, personality changes, and memory loss within 1 to 3 days after surgery. According to reports, the incidence of POCD within 1 week after major non-cardiac surgery in elderly patients is as high as $25.8 \%$ (2). Thus, POCD prevention has become a research hotspot, and there is currently no unified view on the impact of the depth of anesthesia on POCD.

This complication not only affects the patient's ability to take care of themselves, but also imposes a great burden on the family and society. As our society enters the aging stage of the population, the number of operations for elderly patients is gradually increasing. Thus, POCD prevention has become a research hotspot. Narcotrend monitoring is a novel anesthesia depth monitoring method that can subdivide the general anesthesia state into six stages and 15 sub-levels of electroencephalogram (EEG) [Narcotrend stage (NTS)]. Among them, NTS D0-D2 (normal anesthesia state), NTS E0-E2 (deep anesthesia state) are suitable for general anesthesia. The appropriate anesthesia depth can have the least impact on the postoperative cognitive function of the patient.

This study aims to systematically evaluate the effects of Narcotrend monitoring of different depths of anesthesia on POCD, in order to provide a reference for clinical applications and further research. Studies have shown that maintaining an intraoperative Bispectral Index (BIS) value of $30-40$ is more conducive to the recovery of early postoperative cognitive function. In this study, a metaanalysis was conducted to systematically evaluate the impact of different BIS values on POCD, in order to provide a clinical reference (3). We present the following article in accordance with the PRISMA reporting checklist (available at https://atm.amegroups.com/article/view/10.21037/atm$22-90 / \mathrm{rc})$.

\section{Methods}

\section{Included data}

We performed a literature search for studies comparing Narcotrend monitoring under different BIS conditions, in order to compare the incidence of early POCD in different patients. The treatment group received deep anesthesia surgery, while the control group received light anesthesia surgery.

The inclusion criteria were as follows: (I) patients aged $>18$ years old, without serious heart, liver, lung, nephropathy, or endocrine disease, and no history of neuropsychiatry or anesthetic allergy; (II) patients with normal cognitive function before surgery who were planning to undergo elective surgery; and (III) postoperative patients with an incidence of early POCD from 1 to 7 days. The exclusion criteria were as follows: (I) studies that did not provide a specific number of cases of cognitive dysfunction; and (II) studies that involved significant differences in the baseline data between the groups (4).

\section{Detection methods}

We performed literature searches of the PubMed, Embase, OVID, Chinese Biomedical Literature Database (CBM), China National Knowledge Infrastructure (CNKI), Wanfang, and VIP databases from the date of establishment of the database to December 2020. The English search terms included depth of anesthesia, POCD, cognitive function, cognitive dysfunction, cognitive impairments, mental disorders, and cognitive performance. The Chinese search terms included depth of anesthesia, and postoperative cognition. There was no language restriction. The references of the retrieved documents were tracked, and the documents that met the inclusion criteria were included $(5,6)$.

PubMed is the industry's most used medical research database, offering the full text of more than 2,300 journals, including the full text of more than 1,100 peer-reviewed titles. Embase contains more than 1,194,000 records and links to more than 100,000 full-text documents in Embase's collection. Embase offers more than 2,300 full- 
text reference books, encyclopedias and non-fiction books, full texts from more than 130 leading historical journals, more than 61,100 historical documents, 66,000 biographies of historical figures, and more than 110,200 historical photographs and maps. OVID, CBM, CNKI contains the full text of 1,750 general reference publications, the full text of which dates back to 1975. Wanfang, and VIP databases provide authoritative medical information on medicine, nursing, dentistry, veterinary medicine, healthcare systems, preclinical science, and others, with more than 4,800 biomedical journals.

\section{Data extraction}

Two reviewers independently extracted data and evaluated the quality of the obtained literature. Differences of opinion were resolved by a third-party ruling. The following data was extracted: (I) general information of the study, such as the first author and year of publication; (II) research methods (retrospective research, etc.); (III) general information of the research subjects, such as the age, gender, and American Society of Anesthesiologists (ASA) classification of the patient; (IV) treatment of the research object, such as the specific anesthesia methods; and (V) research results [the evaluation method of the research outcome, the research results (four-grid table data), etc.].

The Jadad scale was used to evaluate the quality of the included literature. The scoring standard: randomization (0-2 points: 0 points for non-description, 1 point for random descriptions only, 2 points for random description of normal methods); blinding method ( $0-2$ points: 0 points for not stated, 1 point for blinding only, 2 points for doubleblind description); and loss to follow-up (including reasons, 0 or 1 point: 0 points for not stated, 1 point for narration). Literature with a score of 7 and a total score of 24 was considered high-quality research (7).

\section{Statistical analysis}

Statistical analysis was performed using Rev-Man 5.3 statistical software provided by the Cochrane Collaboration. Measurement data were expressed in terms of the weighted mean difference (WMD) and its 95\% confidence interval (CI), and count data were expressed in terms of odds ratio (OR) and its 95\% CI. Subgroup and statistical homogeneity analyses were also performed, with $\mathrm{P}>1$ as the homogeneity test level. When the $\mathrm{Q}$ statistic of the heterogeneity test was $P>0.1$, it was considered that there was no obvious heterogeneity between the studies, and the fixed-effects model was used for analysis. However, if the heterogeneity test statistic $\mathrm{P}>0.1$, it was considered that there was heterogeneity between the studies. In these cases, the sources of heterogeneity were divided into subgroups, and a random effects model was used (8).

\section{Results}

\section{Retrieval results and quality evaluation}

A total of 534 documents were obtained from the initial search, including 114 from PubMed, 35 from OVID, 117 from CNKI, 97 from CBM, 137 from Wanfang, and 34 from VIP (Figure 1). After carefully reading the abstracts and full texts, a total of eighty studies with a total 714 patients met the inclusion criteria. The basic data of the included studies are shown in Table 1.

\section{The influence of preoperative chemotherapy and different anesthesia methods on hemorheology indexes of cancer patients}

During epidural anesthesia, due to the expansion of blood vessels in the blocking area, the blood vessel resistance decreases, the blood flow accelerates, the blood is diluted, the red blood cell aggregation decreases, and the blood hematocrit decreases, resulting in a decrease in the viscosity of the whole blood. At the same time, epidural anesthesia can also improve fibrinolytic function, improve the interaction between white blood cells, platelets, red blood cells and plasma proteins, and reduce the incidence of thromboembolism. The local anesthetic lidocaine degrades fibrinogen, reducing the surface bridging of red blood cells and making it difficult for red blood cells to accumulate. Procaine can cause spheroidization of erythrocytes, increased red blood cell aggregation and decreased deformability. Isoflurane, halothane, procaine, and ketamine can change the membrane of erythrocytes and reduce the ability of red blood cells to deform. When intravenous injections of haloperidol, fentanyl, diazepam, and thiopental sodium, a decrease in blood viscosity and an increase in the ability of red blood cells to deform can be observed. It is currently believed that intravenous anesthesia with scopolamine hydrobromide trihydrate can form a stable group through its aromatic ring and free radical reaction, such as the combination of phenoxy radicals of propofol and free radicals, inhibit voltage-dependent calcium channels, reduce intracellular calcium overload 


\section{Identification of studies via databases and registers}

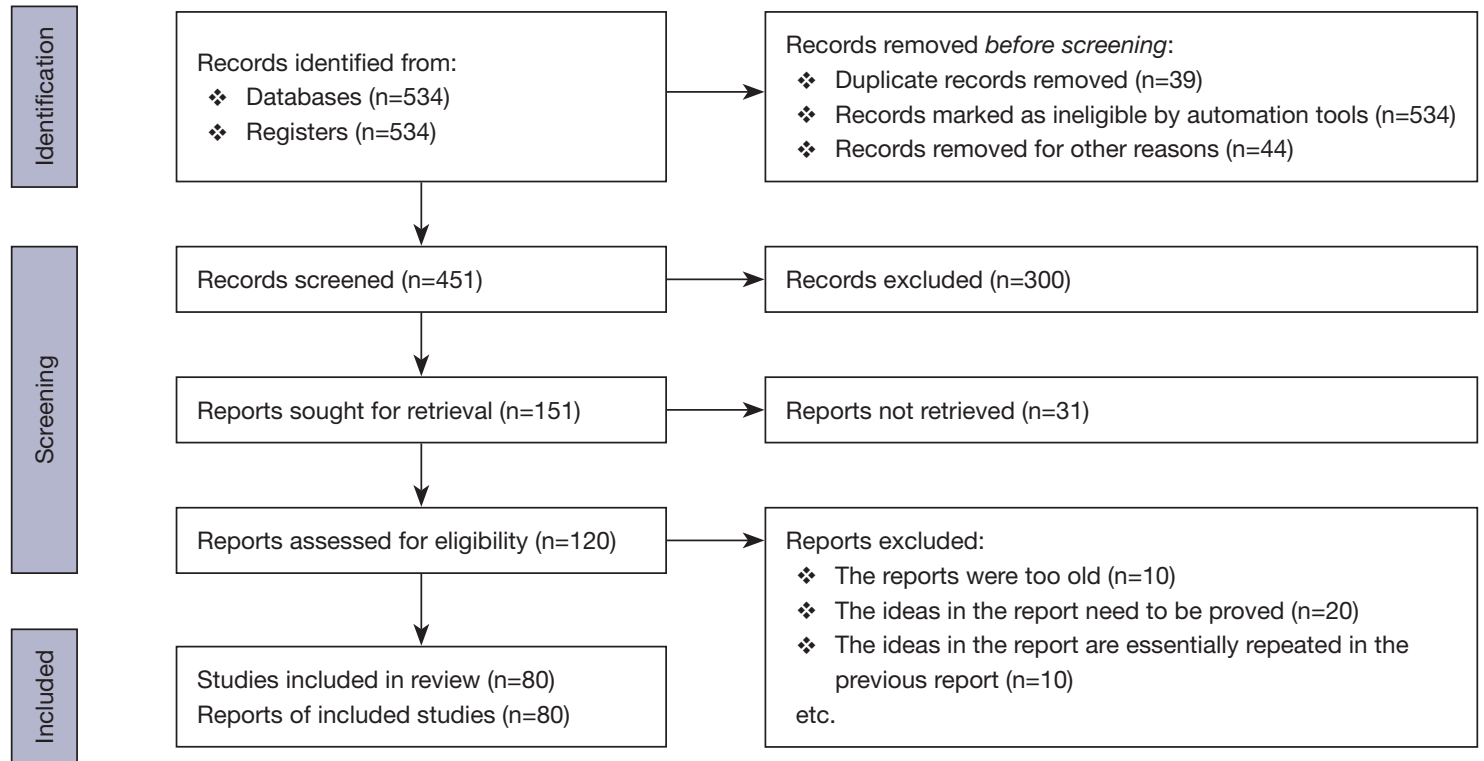

Figure 1 PRISMA flowchart.

and thus inhibit the production of free radicals, show dosedependent delay, and reduce the process of mitochondrial lipid peroxidation, which can protect red blood cells in the human body. At the same time, studies have found that (IV), $1-1.5 \mathrm{mg} / \mathrm{kg}$ propofol can significantly reduce hematocrit and maintain it for 48 hours, therefore, after general anesthesia, it can reduce the effect of catecholamines and improve blood rheology. This change favors the perfusion of blood flow to tissues and organs. Inhalation of high concentrations of oxygen and inhalation of narcotics is still potentially dangerous for the ability to deform red blood cells. Epidural anesthesia is a better anesthesia for patients with preoperative erythrocyte deformation, which can significantly reduce the incidence of deep vein thrombosis and pulmonary thrombosis, especially lower extremity surgery.

\section{Meta-analysis results}

A total of three studies compared the incidence of POCD on the first day after deep anesthesia (NTS E0-E1) and conventional anesthesia (NTS D0-D1). There was no significant heterogeneity between the studies $(\mathrm{P}=0.94$, $\mathrm{I}^{2}=0 \%$ ), so a fixed effects model was used for meta-analysis $(17,18)$. The results showed that the incidence of POCD on the first day after deep anesthesia was significantly lower than that of conventional anesthesia (OR $=0.21,95 \% \mathrm{CI}$ : 0.13-0.35, $\mathrm{P}<0.00001$ ) (Figure 2).

Compared with conventional anesthesia (NTS D0), the incidence of POCD at 7 days postoperatively, there was no heterogeneity among the studies $\left(\mathrm{P}=0.94, \mathrm{I}^{2}=0 \%\right)$, and the fixed effects model was used for meta-analysis. The results showed that the incidence of POCD 7 days after deep anesthesia was significantly lower than that of conventional anesthesia (OR $=0.45,95 \%$ CI: 0.23-0.91, $\mathrm{P}=0.03)$ (Figure 3).

A total of five studies compared the incidence of POCD 7 days after surgery of NTS D2 and NTS D0 in conventional anesthesia. There was no heterogeneity among the studies $\left(\mathrm{P}=0.87, \mathrm{I}^{2}=0 \%\right)$, and thus the fixed effects model was used for meta-analysis. The results showed that NTS POCD incidence of postoperative NTS D2 group was significantly lower than NTS D0 group (OR $=0.42,95 \%$ CI: $0.24-0.71$, $\mathrm{P}=0.001$ ) (Figure 4).

\section{Optimization of intraoperative anesthesia depth and regional brain oxygenation can reduce postoperative cognitive decline in elderly patients}

POCD is one of the more common complications after surgery, and most patients present with memory loss, 
Table 1 Basic characteristics of the included studies

\begin{tabular}{|c|c|c|c|c|c|c|}
\hline Study & Age & ASA rating & Type of surgery & Research design & $\begin{array}{l}\text { Detection } \\
\text { indicator }\end{array}$ & Detection time \\
\hline Le, 2014, (9) & $>60$ years old & N-M level & $\begin{array}{l}\text { General anesthesia } \\
\text { abdominal surgery }\end{array}$ & Random number table & (I) & $\begin{array}{l}\text { Preoperative } 1 \text { day, } \\
\text { after } 1 \text { day }\end{array}$ \\
\hline Wu, 2015, (10) & $>70$ years old & I-M level & $\begin{array}{l}\text { Laparoscopic radical } \\
\text { resection of bowel cancer }\end{array}$ & $\begin{array}{l}\text { Random (unknown } \\
\text { method) double blind }\end{array}$ & $\begin{array}{l}\text { (I) (II) (III) } \\
\text { (IV) (V) }\end{array}$ & $\begin{array}{l}7 \text { days before and } \\
\text { after operation }\end{array}$ \\
\hline Feng, 2016, (12) & $\geq 60$ years old & $\mathrm{N}-\mathrm{M}$ level & $\begin{array}{l}\text { General anesthesia } \\
\text { abdominal surgery }\end{array}$ & Random number table & (l) & $\begin{array}{l}\text { Before operation and } \\
1 \text { day after operation }\end{array}$ \\
\hline Kang, 2018, (13) & $60-92$ years old & I-M level & $\begin{array}{l}\text { Laparoscopic radical } \\
\text { resection of bowel cancer }\end{array}$ & $\begin{array}{l}\text { Computer Random } \\
\text { Number Method }\end{array}$ & $\begin{array}{l}\text { (I) (II) (III) } \\
\text { (IV) (V) }\end{array}$ & $\begin{array}{l}1 \text { d before operation, } \\
7 \text { days after } \\
\text { operation }\end{array}$ \\
\hline Deng, 2016, (15) & $>65$ years old & $\mathrm{I}-\mathrm{N}$ level & Abdominal surgery & $\begin{array}{l}\text { Random (method } \\
\text { unknown) }\end{array}$ & (I) $(\mathrm{VI})$ & $\begin{array}{l}1 \text { day before } \\
\text { operation, } 7 \text { days } \\
\text { after operation }\end{array}$ \\
\hline Gao, 2014, (16) & 3-65 years old & $\mathrm{I}-\mathrm{N}$ level & $\begin{array}{l}\text { Upper abdominal general } \\
\text { surgery }\end{array}$ & $\begin{array}{l}\text { Random (method } \\
\text { unknown) }\end{array}$ & (I) $(\mathrm{VI})$ & $\begin{array}{l}1 \text { day before } \\
\text { operation, } 7 \text { days } \\
\text { after operation }\end{array}$ \\
\hline
\end{tabular}

A total of three studies compared the state of deep anesthesia (NTSE1). (I) Simple mental state checklist MMSE; (II) number span test; (III) number symbol test; (IV) tracking line test; (V) word fluency test; (VI) quantitative Montreal cognitive assessment. ASA, American Society of Anesthesiologists.

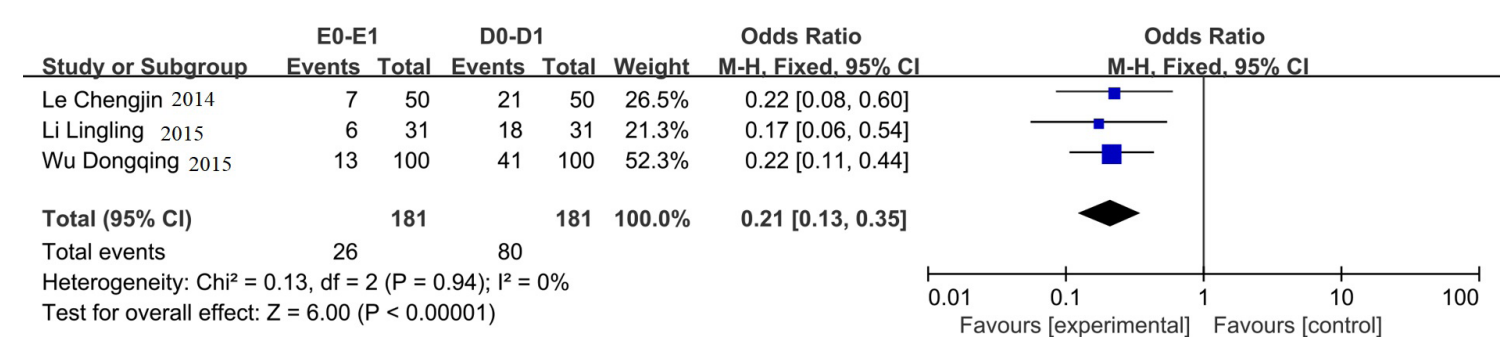

Figure 2 Forest plot of POCD incidence on day 1 after deep anesthesia and conventional anesthesia. POCD, postoperative cognitive dysfunction; M-H, Mantel-Haenszel test; CI, confidence interval; Chi ${ }^{2}$, chi-squared statistic; df, degree of freedom; $\mathrm{I}^{2}$, percentage of total variation in effect size between studies due to heterogeneity rather than sampling error; $\mathrm{Z}$, chi-square value of heterogeneity test.

long duration, and even permanent damage, which increases the burden on families and society. Studies have shown that POCD is caused by multifactorial influences, including the choice of anesthesia modalities, the age of the patient, the duration of anesthesia, the amount of intraoperative bleeding and the amount of rehydration fluids Intraoperative hypotension caused by hypoperfusion of brain tissue, etc. In particular, it is crucial to maintain a moderate depth of anesthesia during the perioperative period, and excessively deep anesthesia is not only detrimental to patient recovery, but may also threaten the patient's life; Too shallow anesthesia will affect the surgical operation, and even occur during the patient's intraoperative awakening, causing mental trauma. The dual-spectrum 


\begin{tabular}{|c|c|c|c|c|c|c|c|c|c|c|}
\hline Study or Subgroup & $\begin{array}{r}\text { E1 } \\
\text { Events }\end{array}$ & Total & $\begin{array}{r}\text { D0 } \\
\text { Events }\end{array}$ & Total & Weight & $\begin{array}{l}\text { Odds Ratio } \\
\text { M-H. Fixed, } 95 \% \mathrm{Cl}\end{array}$ & & $\begin{array}{r}\text { Odds } \\
\text { M-H. Fixe }\end{array}$ & $\begin{array}{l}\text { Ratio } \\
\text { ed. } 95 \% \mathrm{Cl}\end{array}$ & \\
\hline Feng Tao 2016 & 4 & 20 & 4 & 10 & $18.0 \%$ & $0.38[0.07,2.00]$ & & & & \\
\hline Kang Yin 2018 & 11 & 49 & 19 & 48 & $62.9 \%$ & $0.44[0.18,1.07]$ & & - & & \\
\hline Liu Nian 2015 & 4 & 16 & 6 & 16 & $19.0 \%$ & $0.56[0.12,2.54]$ & & & & \\
\hline Total $(95 \% \mathrm{Cl})$ & & 85 & & 74 & $100.0 \%$ & $0.45[0.23,0.91]$ & & & & \\
\hline Total events & 19 & & 29 & & & & & & & \\
\hline $\begin{array}{l}\text { Heterogeneity: } \mathrm{Chi}^{2}= \\
\text { Test for overall effect: }\end{array}$ & $\begin{array}{l}12, \mathrm{df}=2 \\
=2.24(\mathrm{P}\end{array}$ & $\begin{array}{l}(P=0 \\
=0.03\end{array}$ & (94); $\left.\right|^{2}=$ & & & & 0.01 & $\begin{array}{ll}0.1 & 1 \\
\text { Favours [E1] }\end{array}$ & $\begin{array}{lr}1 & 10 \\
\text { Favours [D0] }\end{array}$ & 100 \\
\hline
\end{tabular}

Figure 3 Forest plot of the incidence of POCD at 7 days after deep anesthesia and conventional anesthesia. POCD, postoperative cognitive dysfunction; M-H, Mantel-Haenszel test; CI, confidence interval; Chi ${ }^{2}$, chi-squared statistic; df, degree of freedom; $\mathrm{I}^{2}$, percentage of total variation in effect size between studies due to heterogeneity rather than sampling error; Z, chi-square value of heterogeneity test.

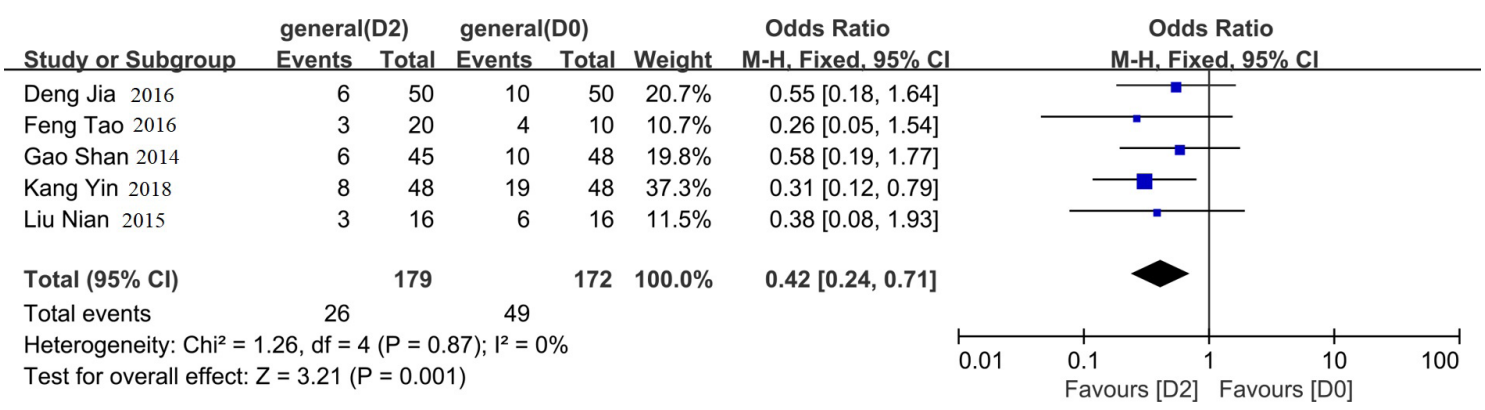

Figure 4 Forest plot of POCD incidence at $7 \mathrm{~d}$ after NTS D2 and NTS D0. NTS, negative correlation is currently the most appropriate egg description; Nd can subdivide the original EEG into six stages 15 levels (Nd Sg, NTS), or A (state of wakefulness), state B0-B2 (sedated state), state C0-C2 (light anesthetic state), state D0-D2 (general anesthesia), state E0-E2 (deep anesthesia state), and state F0-F2 (burst suppression state); POCD, postoperative cognitive dysfunction; M-H, Mantel-Haenszel test; CI, confidence interval; Chi ${ }^{2}$, chi-squared statistic; df, degree of freedom; $\mathrm{I}^{2}$, percentage of total variation in effect size between studies due to heterogeneity rather than sampling error; $Z$, chi-square value of heterogeneity test.

index is the result of the comprehensive analysis of the spectral boundary frequency and intermediate frequency in EEG activities, and is currently used in clinical practice. BIS is currently the longest, most mature and most widely used method of in-depth monitoring of anesthesia in the clinic, with a dual-spectrum index range of 0 to 100 , and the smaller the index, the deeper the anesthesia. It is shown that maintaining the bis value between 30 and 39 can promote the recovery of postoperative cognitive function and reduce the occurrence of cognitive dysfunction. The reason may be that the BIS value maintained between 30 and 39 has less impact on central neurotransmitters and receptor systems, and can also promote the proliferation of some nerve cells.

Application in surgery. A recent study in patients undergoing oncology showed a decrease in local oxygen saturation $\mathrm{rSO} 2$ and POCD (POST operative cognitive dysfunction). Local $\mathrm{rSO} 2$ monitoring predicts the sensitivity and specificity of POCD, respectively $8 \%$ and
$85 \%$. However, older patients with medullary fractures can develop cognitive dysfunction both before and after surgery and are associated with low $\mathrm{rSO} 2$ values. A recent study in patients on shoulder arthroscopy under a beach chair position showed that intraoperative $\mathrm{rSO} 2$ reduction was not associated with POCD, but they speculated that monitoring rSO2 can help to decrease the occurrence of postoperative cognitive dysfunction and improves patient safety.

\section{Sensitivity analysis}

Sensitivity analysis was carried out by changing the model, removing the maximum weight, and removing the minimum weight literature. The results showed that after three changes, the result has little change from the original OR value. Therefore, it can be considered that the sensitivity was low, and the results of this study were relatively stable and basically reliable. 
Table 2 Methodological quality evaluation of the included studies

\begin{tabular}{|c|c|c|c|c|c|}
\hline Study & Random method & Blinding & Allocation hiding & Lost to follow-up/exit & Improved Jadad score \\
\hline Hao, 2014, (20) & Not sure & Not sure & Not sure & Without & 3 \\
\hline Bian, 2014, (21) & $\begin{array}{l}\text { Computer Random } \\
\text { Number Method }\end{array}$ & Not sure & Not sure & Without & 4 \\
\hline
\end{tabular}

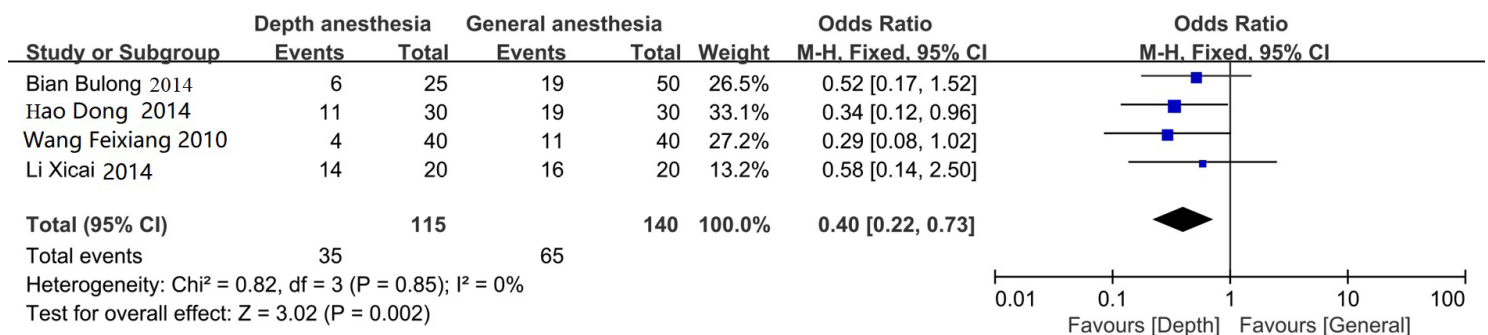

Figure 5 Comparison of the incidence of POCD on the first postoperative day between deep anesthesia and conventional anesthesia. POCD, postoperative cognitive dysfunction; M-H, Mantel-Haenszel test; CI, confidence interval; Chi ${ }^{2}$, chi-squared statistic; df, degree of freedom; $\mathrm{I}^{2}$, percentage of total variation in effect size between studies due to heterogeneity rather than sampling error; $Z$, chi-square value of heterogeneity test.

In the end, six studies met the inclusion criteria, including five Chinese studies and one English article, with a total of 521 patients. Four studies did not describe the method of random sequence generation, five studies did not describe the hiding of randomized sequences, and none of the studies described whether blinding was used, however, the subjects were blinded (i.e., single-blind) (Table 2). Furthermore, all of the included studies described the reasons for loss to follow-up as well as the treatment of these cases, and it could be considered that there is no incomplete data bias. The methodological quality evaluation of the included studies was detailed in four studies comparing the incidence of POCD on the first day after deep anesthesia (BIS 30-40) and conventional anesthesia (BIS 40-60). There was no heterogeneity among the studies, and the fixed effects model was used for analysis. The results showed that under deep anesthesia, the incidence of POCD was significantly lower than that under conventional anesthesia $(\mathrm{OR}=0.40$, 95\% CI: 0.22-0.73, $\mathrm{P}=0.002$ ) (Figure 5) (24).

The incidence of early postoperative POCD was compared between BIS 40-50 and BIS 50-60 in conventional anesthesia. There was heterogeneity among the research results, and thus, the random effects model was used for analysis (25). The results showed that there were no statistically significant differences in the incidence of early postoperative POCD between BIS 40-50 and BIS 50-60 (OR =1.11, 95\% CI: 0.24-5.24, $\mathrm{P}=0.90)$ (Figure 6).

The deep anesthesia state (BIS 30-40) and the conventional anesthesia state (BIS 40-60) were compared on the third day after surgery, the incidence of POCD. There was no obvious heterogeneity between the studies, and the fixed-effects model was used for analysis. The results showed that after the first three deep anesthesia in anesthetic-day conventional POCD difference in the incidence of no statistical significance $(\mathrm{OR}=0.47,95 \% \mathrm{CI}$ : 0.13-1.71, $\mathrm{P}=0.25$ ) (Figure 7) (26).

\section{Discussion}

At present, the pathogenesis of POCD is not clear. It may be based on the aging of the nervous system, which is induced or aggravated by adverse stresses such as anesthesia and surgical trauma. Although the depth of anesthesia may have a certain relationship with the occurrence of POCD, there is currently no unified view. The intervention measures of this article are to perform anesthesia and 


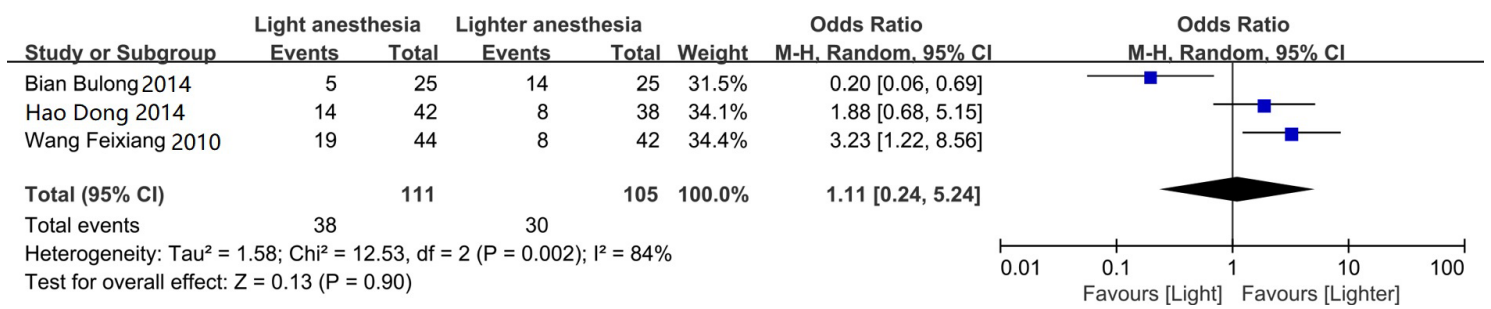

Figure 6 Comparison of the incidence of POCD in early postoperative period between BIS 40-50 and BIS 50-60. BIS, Bispectral Index; POCD, postoperative cognitive dysfunction; M-H, Mantel-Haenszel test; CI, confidence interval; Tau ${ }^{2}$, size of heterogeneity; Chi ${ }^{2}$, chisquared statistic; df, degree of freedom; $\mathrm{I}^{2}$, percentage of total variation in effect size between studies due to heterogeneity rather than sampling error; $Z$, chi-square value of heterogeneity test.

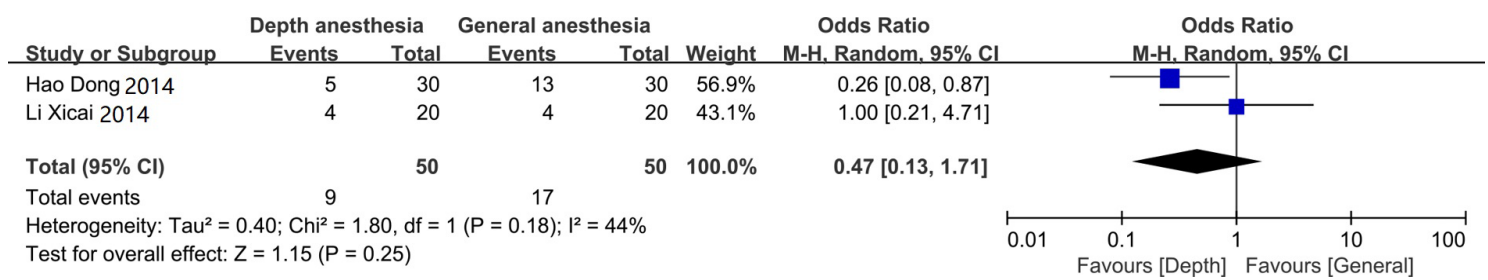

Figure 7 Comparison of the incidence of POCD between BIS 30-40 and BIS 40-60 on postoperative day 3. BIS, Bispectral Index; POCD, postoperative cognitive dysfunction; M-H, Mantel-Haenszel test; CI, confidence interval; Tau ${ }^{2}$, size of heterogeneity; Chi ${ }^{2}$, chi-squared statistic; df, degree of freedom; $\mathrm{I}^{2}$, percentage of total variation in effect size between studies due to heterogeneity rather than sampling error; $Z$, chi-square value of heterogeneity test.

surgical operations on patients, so it is difficult to doubleblind the study subjects (27).

In this study, six articles with different BIS values were systematically evaluated, and the effects of different BIS values on the incidence of early postoperative POCD were compared. Four studies compared deep anesthesia (BIS 30-40) and general anesthesia (BIS 40-60) after the first day. At 1 day postoperatively, the incidence of POCD was significantly lower under general anesthesia than under deep anesthesia.

Elderly patients are prone to cognitive function impairment after surgery due to degenerative changes in the structure and function of tissues and organs, progressive decline in organ reserve function, prolonged drug metabolism time, and preoperative complications with other underlying diseases. Delirium (POCD) can lead to severe delays in recovery, prolonged hospital stays, long-term cognitive dysfunction, and potential increased mortality. The vast majority of cognitive impairments are reversible, but a small number of patients may remain with long-term or permanent cognitive impairment. At present, delirium can be divided into 3 types: the main type is the positive symptom increase activity type, the negative symptom mainly decreased activity type, and the mixed type. At present, there are no biological indicators or imaging tests that can be fully relied upon to confirm the diagnosis of delirium, and in some cases, when there are typical symptoms of hyperactivity, the diagnosis of delirium is mainly based on subjective clinical impressions, but subjective diagnosis leads to a high rate of misdiagnosis of activity-increasing delirium and the susceptibility to missed delirium, and studies have shown that the missed diagnosis rate can be as high as $66 \%$.

Systematic analysis of conventional anesthesia states such as BIS 40-50 and 50-60 showed that there was heterogeneity between the included studies. After analyzing and processing the existing heterogeneity, the results showed that there was no significant difference in the incidence of POCD under conventional anesthesia. This may be due to different studies using different surgical methods and anesthesia protocols. In addition, the sample size of this study is too small, which leads to deviations in the results.

This study further compared the incidence of POCD on the 3rd day after surgery under deep anesthesia (BIS 30-40) and conventional anesthesia (BIS 40-60), and the 
results showed that there was no significant difference between the two methods. This result may be compared with the literature method included in the study; poor learning quality is related to factors such as small sample size. Therefore, it is necessary to design more large-sample and high-quality randomized controlled trials for further systematic analysis.

In addition, this study found that under deep anesthesia, such as when BIS was 30-40, the incidence of POCD in the early postoperative period was lower than that of conventional anesthesia, which is consistent with the research conclusions. Previous studies have shown that deep anesthesia can significantly reduce the cerebral oxygen metabolism rate, thereby reducing the incidence of POCD in patients. Other studies have shown that deep anesthesia can inhibit the levels of cortisol, epinephrine, norepinephrine, etc., and reduce the body's stress response (28).

In summary, deep anesthesia has a certain effect on reducing POCD in the early postoperative period. However, due to the lack of multi-center studies in the literature included in this study, as well as the insufficient number and quality of randomized controlled trials, it was impossible to compare the incidence on the third or even the seventh day after surgery. Therefore, it is necessary to further develop rigorously designed and high-quality randomized controlled trials (29).

POCD is the result of a combination of multiple factors, including the preoperative application of anticholinergic drugs, the patient's age, the preoperative underlying disease, the type of operation, the method and duration of anesthesia, the depth of anesthesia, as well as intraoperative hypotension and hypoxia etc. Older age is a definite influencing factor for the occurrence of POCD; the older the age, the higher the incidence of POCD and the longer the duration. The application of Narcotrend to select an appropriate depth of anesthesia is more conducive to reducing the incidence of various adverse reactions. Therefore, more attention should be paid to the influence of different depths of anesthesia on POCD (30).

Clinical BIS monitoring involves two different components of anesthesia regimen-hypnosis and analgesia. This concept makes it necessary to regulate the dosage of hypnotic and analgesics, such as midazolam, propofol, isoflurane, and sevoflurane, to maintain lower levels of BIS between 40 and 60 . This range appears to be a therapeutic window for basic unconscious states. When the manual stimulation is intense, if the BIS index rises and the patient develops a kinetic and hemodynamic response, the anesthesiologist should increase the hypnotic component to reduce the BIS value to 40 to 60 . If the BIS value is between 40 and 60 and the patient still has a systemic and hemodynamic response, opioids should be added continuously to increase the analgesic component of the anesthesia until the body movement and hemodynamic response are controlled; when the anesthesia is about to end, the hypnotic component should be reduced to allow the BIS index to rise. If general anesthesia is induced, only propofol reduces the BIS value to 45 to 55 . The fluctuations in the mechanics of blood flow in patients are controlled by vasoactive drugs, and the results are worth studying.

Narcotrend Anesthesia Trend is a new type of EEG/ consciousness depth monitoring system with a professional EEG signal collection amplifier, which can accurately collect, monitor, and analyze real-time EEG signals at any position of the brain through ordinary ECG electrodes. Studies in China and abroad have found that Narcotrend can accurately reflect the changes in the depth of anesthesia during intravenous and inhalation anesthesia, and the depth of anesthesia is negatively correlated with NTS, which is currently the most suitable description for EEG (31).

Narcotrend can subdivide the original EEG into six stages and 15 levels (NTS), namely A (awake state), state B0-B2 (sedation state), state C0-C2 (light anesthesia state), state D0-D2 (conventional anesthesia state), state E0-E2 (deep anesthesia state), and state F0-F2 (burst suppression state), and use the 0-100 dimensionless anesthesia depth index [Narcotrend index (NI)] to reflect the whole process from awake to deep anesthesia. Compared with BIS, Narcotrend not only distinguishes the state of consciousness over a large span, but also has provides the advantage of monitoring sudden changes in the depth of anesthesia (32).

This study included eight articles and compared the effects of Narcotrend monitoring of different depths of anesthesia on POCD. The results showed that the incidence of early postoperative POCD under Narcotrend monitoring of deep anesthesia (E0-E1) was significantly lower than that of conventional anesthesia (D0-D1). The depth of anesthesia was maintained at the level of NTS D2 under conventional anesthesia. Compared with NTS D0, the incidence of postoperative POCD was significantly reduced (33).

Whether anesthesia has adverse effects on cognitive function is an area of particular interest for clinical anesthesiologists. The mechanism of deep anesthesia to protect cognitive function remains unclear (34). It may be that deep anesthesia reduces brain metabolism and 
produces neuroprotective effects, which further reduces the incidence of POCD in patients. In addition, surgery to stimulate the body's release of inflammatory factors and activation of glial cells can cause POCD cold. Studies such as FIDALGO have shown that an appropriate depth of anesthesia can also inhibit the inflammatory stress response and help protect the brain. However, in recent years, there are limitations and controversies in the monitoring of the depth of anesthesia, and there are still reports of awakening of patients under deep anesthesia. Therefore, this requires further study.

In this study, from the experimental results, whether the maximum or median value was selected for statistical analysis. It can distinguish between coma and vegetative state, and the BIS values of comatose patients are significantly smaller than those in VS patients, and VS and minimally conscious state can also be distinguished.

Previous articles have mostly only mentioned the correlation between BIS values and Glasgow Coma Scale (GCS) scores, as far as BIS values are related to CRS-R values There are very few articles on it, and BIS values and CRS-R values are proposed for the first time in the article BIS can be used to determine the two levels of consciousness of plant state and micro-conscious state, which is consistent with the point of view of this study (35).

The relationship, the GCS also increases, and the corresponding BIS value also increases; and bis and CRS-R are also positively correlated, but when BIS is more relevant to GCS than BIS to CRS-R. The correlation between BIS and CRS-R is higher than that obtained when BIS is the maximum. The BIS value of the VS group is significantly lower than that of the micro-consciousness state, so BIS can be used to distinguish VS and MCS. According to the statistical analysis of the data, the BIS values of the coma and VS groups were: 57 (21.5) and 73 (24.0), after statistical analysis, $\mathrm{P}<0.5$, the BIS value in comatose patients was significantly lower than that in VS patients, and no correlation was seen in the past.

For the first time, this study has found that BIS can be used as an objective indicator to distinguish between coma and VS. However, it needs to be confirmed by further multicenter and large sample size research.

There is a close relationship between the state and the high level.

This systematic review has certain limitations that should be noted. At present, there are few studies on Narcotrend monitoring the influence of different depths of anesthesia on POCD in China and abroad (36). Due to the high literature requirements for meta-analysis, only eight articles met the final inclusion criteria. The sample sizes and the numbers of studies are insufficient, and thus, more large-sample and high-quality multi-center studies and randomized controlled trials need to be designed for further systematic analysis. In addition, due to the particularity of the anesthesia and surgeries to be performed on patients, it was difficult to implement rigorous blinding of the study subjects. The inclusion criteria did not report the allocation concealment, so the results of this study should be cautiously interpreted and applied (37).

In conclusion, deep anesthesia under Narcotrend monitoring can reduce the occurrence of early postoperative POCD, which can provide certain evidence-based medical assistance for the clinical work of anesthesiologists. Thus, deep anesthesia has a certain mitigating effect on POCD in the early postoperative period. However, the literatures included in this study lack multi-center research, as well as comparison of the incidences on postoperative days 3 or even 7, and therefore, more well-designed, high-quality controlled trials are needed to validate our findings (38).

\section{Acknowledgments}

Funding: None.

\section{Footnote}

Reporting Checklist: The authors have completed the PRISMA reporting checklist. Available at https://atm. amegroups.com/article/view/10.21037/atm-22-90/rc

Conflicts of Interest: All authors have completed the ICMJE uniform disclosure form (available at https://atm. amegroups.com/article/view/10.21037/atm-22-90/coif). The authors have no conflicts of interest to declare.

Ethical Statement: The authors are accountable for all aspects of the work in ensuring that questions related to the accuracy or integrity of any part of the work are appropriately investigated and resolved.

Open Access Statement: This is an Open Access article distributed in accordance with the Creative Commons Attribution-NonCommercial-NoDerivs 4.0 International License (CC BY-NC-ND 4.0), which permits the noncommercial replication and distribution of the article with the strict proviso that no changes or edits are made and the 
original work is properly cited (including links to both the formal publication through the relevant DOI and the license). See: https://creativecommons.org/licenses/by-nc-nd/4.0/.

\section{References}

1. Shen JH, Ye M, Chen Q, et al. Effects of circadian rhythm on Narcotrend index and target-controlled infusion concentration of propofol anesthesia. BMC Anesthesiol 2021;21:215.

2. Hight D, Schanderhazi C, Huber M, et al. Age, minimum alveolar concentration and choice of depth of sedation monitor: examining the paradox of age when using the Narcotrend monitor: A secondary analysis of an observational study. Eur J Anaesthesiol 2021. [Epub ahead of print].

3. Heiderich S, Ghasemi T, Dennhardt N, et al. Correlation of exhaled propofol with Narcotrend index and calculated propofol plasma levels in children undergoing surgery under total intravenous anesthesia - an observational study. BMC Anesthesiol 2021;21:161.

4. Raue JF, Tünsmeyer J, Kästner SBR. Effects of isoflurane, remifentanil and dexmedetomidine on selected EEG parameters derived from a Narcotrend Monitor before and after nociceptive stimulation at different MAC multiples in cats. BMC Vet Res 2020;16:332.

5. Puchner WF, Dünser MW, Paulus P, et al. A comparative study on adequate anesthesia depth: clinical judgement and the Narcotrend ${ }^{\circledR}$ measurement. Can J Anaesth 2020;67:664-73.

6. Villa EK, Villa D, Bundoc RC. Narcotrend-guided intraoperative care of a Trisomy 21 paediatric patient who underwent occipitocervical fusion. BMJ Case Rep 2020;13:231276.

7. de Heer IJ, Warmenhoven AT, Weber F. Electroencephalographic density spectral array monitoring during propofol sedation in teenagers, using the narcotrend electroencephalographic monitor. Minerva Anestesiol 2020;86:601-7.

8. Plaschke K, Weiskircher A, Benner L, et al. Depth of anesthesia by Narcotrend $\AA$ and postoperative characteristics in children undergoing cardiac surgery under extracorporeal circulation: a retrospective comparison of two anesthetic regimens. Perfusion 2020;35:427-35.

9. Le CJ. Effects of different anesthesia management methods on s100-P protein level and cognitive function in elderly patients. Chinese Journal of Laboratory Diagnosis
2014;18:912-5.

10. Wu DQ, Xue JP, Ding Q, et al. Effects of different depth of anesthesia on postoperative cognitive impairment in elderly patients undergoing abdominal surgery. Chinese and Foreign Medical Research 2015;13:40-1.

11. Liu N. Narcotrend monitoring the effect of different anesthesia depth on early postoperative cognitive impairment in elderly patients with colorectal cancer. Contemporary Medicine 2015;21:82-3.

12. Feng T. Narcotrend monitoring the effect of different anesthesia depth on early postoperative cognitive impairment in elderly patients with colorectal cancer. The World Clinical Medicine 2016,10:29-31.

13. Kang Y, Deng LJ, Zhao GD, et al. Effect of anesthetic depth on early cognitive dysfunction in elderly patients with colorectal cancer. Journal of Clinical Anesthesiology 2018;29:734-7.

14. Li LL, Luo XY, Chen T, et al. Effects of different depth of anesthesia on postoperative cognitive function and serum S100-P protein level in elderly patients. Medical Journal of West China 2015;27:1352-5.

15. Deng J, Jiang L, Xu T, et al. Effects of depth of anesthesia on postoperative cognitive function in elderly patients. Gansu Medical Journal 2016;35:172-5.

16. Gao S, Zhou N, Chen CL, et al. Influence of dose depth of anesthesia assessed by the Narcotrend index on postoperative cognitive dysfunction in elderly patients after abdominal surgery. Military Medical Journal of Southeast China 2014;16:14-7.

17. Spineli LM, Pandis N. Fixed-effect versus randomeffects model in meta-regression analysis. Am J Orthod Dentofacial Orthop 2020;158:770-2.

18. Zhang M, Yuan J, Chen Q, et al. Application of Narcotrend index and Richmond agitation-sedation score in sedation assessment of patients with short-term mechanical ventilation after pancreatoduodenectomy: a randomized controlled trial. Zhonghua Wei Zhong Bing Ji Jiu Yi Xue 2019;31:737-41.

19. Li XC, Xu KB, Cui G, et al. Depth of anesthesia and postoperative cognitive dysfunction Correlation study of obstruction. Journal of Mudanjiang Medical University 2014;35:94-6.

20. Hao D, Gao Y, Zhang J. Effect of different depth of anesthesia on postoperative cognitive dysfunction in elderly patients the effect of hindering occurrence. Chinese Journal of Anesthesiology 2014;34:251-2.

21. Bian BL, Yin YQ, Sun B, et al. Early postoperative cognitive impairment in elderly patients with LC The 
relationship between obstruction and the change of dual frequency index of electroencephalogram during operation. Chinese Journal of Clinical Research 2014;27:185-7.

22. Wang FX, Yan M. Effects of different depth of general anesthesia on postoperative cognitive function in elderly patients the influence of. Zhejiang Medical Journal 2010;32:397-9.

23. Hirota K, Matsunami K, Kudo T, et al. Relation between bispectral index and plasma catecholamines after oral diazepam premedication. Eur J Anaesthesiol 1999;16:516-8.

24. Dennhardt N, Arndt S, Beck C, et al. Effect of age on Narcotrend Index monitoring during sevoflurane anesthesia in children below 2 years of age. Paediatr Anaesth 2018;28:112-9.

25. Dennhardt N, Beck C, Boethig D, et al. Impact of temperature on the Narcotrend Index during hypothermic cardiopulmonary bypass in children with sevoflurane anesthesia. Perfusion 2018;33:303-9.

26. Kant R, Dubey PK, Ranjan A. Palonosetron Pretreatment is not as Effective as Lignocaine for Attenuation of Pain on Injection of Propofol. Turk J Anaesthesiol Reanim 2020;48:196-201.

27. Dennhardt N, Boethig D, Beck C, et al. Optimization of initial propofol bolus dose for EEG Narcotrend Index-guided transition from sevoflurane induction to intravenous anesthesia in children. Paediatr Anaesth 2017;27:425-32.

28. Tünsmeyer J, Hopster K, Kästner SB. Clinical Use of a Multivariate Electroencephalogram (Narcotrend) for Assessment of Anesthetic Depth in Horses during Isoflurane-Xylazine Anesthesia. Front Vet Sci 2016;3:25.

29. Otto KA. Differential effects of propofol and isoflurane on the relationship between EEG Narcotrend index and clinical stages of anaesthetic depth in sheep undergoing experimental cardiac surgery. Vet J 2016;208:87-9.

30. Ji J, Wang G, Sun K, et al. Application of dexmedetomidine combined with propofol in patients undergoing painless colonoscopy for colonic polyps

Cite this article as: Shi X, Chen X, Ni J, Zhang Y, Liu H, $\mathrm{Xu} \mathrm{C}$, Wang H. Systematic review and meta-analysis of the prognostic value of Narcotrend monitoring of different depths of anesthesia and different Bispectral Index (BIS) values for cognitive dysfunction after tumor surgery in elderly patients. Ann Transl Med 2022;10(4):186. doi: 10.21037/atm-22-90 resection under Narcotrend monitoring. Nan Fang Yi Ke Da Xue Xue Bao 2015;35:264-7.

31. Duan G, Guo S, Zhan H, et al. A new real-time method for detecting the effect of fentanyl using the preoperative pressure pain threshold and Narcotrend index: a randomized study in female surgery patients. Medicine (Baltimore) 2015;94:e316.

32. Qiu YS, Xu Q. Accuracy of narcotrend index in monitoring depth of anesthesia in diabetics: a case report. Chin Med Sci J 2014;29:251-2.

33. Guo Z, Pang L, Jia X, et al. Intraoperative targetcontrolled infusion anesthesia application using remifentanil hydrochloride with etomidate in patients with severe burn as monitored using Narcotrend. Burns 2015;41:100-5.

34. Shepherd J, Jones J, Frampton G, et al. Clinical effectiveness and cost-effectiveness of depth of anaesthesia monitoring (E-Entropy, Bispectral Index and Narcotrend): a systematic review and economic evaluation. Health Technol Assess 2013;17:1-264.

35. Jiang Y, Qiao B, Wu L, et al. Application of Narcotrend® monitor for evaluation of depth of anesthesia in infants undergoing cardiac surgery: a prospective control study. Braz J Anesthesiol 2013;63:273-8.

36. Rinösl H, Fleck T, Dworschak M. Brain ischemia instantaneously tracked by the narcotrend EEG device. J Cardiothorac Vasc Anesth 2013;27:e13-4.

37. Guo ZG, Wang XY, Lü XL, et al. Application of Narcotrend-assisted anesthesia in-depth monitor during escharectomy and skin transplantation in burn patients with target-controlled infusion of remifentanil hydrochloride and propofol. Zhonghua Shao Shang Za Zhi 2012;28:178-82.

38. Li CX, Li H. Determination of the $50 \%$ effective concentration of dexmedetomidine as an adjuvant in combined spinal-epidural anesthesia with Narcotrend. Nan Fang Yi Ke Da Xue Xue Bao 2011;31:734-6.

(English Language Editor: A. Kassem) 